\title{
Neural Network Method Applying in Acid Fracturing Choosing Well of Zanarol Oil Field
}

\author{
Liu Zhenyu \\ Northeast Petroleum University \\ Da Qing, China \\ Email: 1zydqsyxy@163.com
}

He Jinbao

Drilling and Production Technology Research Institute, Instrumentation Research Institute, Liao He Oilfield,

China Petroleum

Pan Jin, China

\author{
Wang Wei \\ Northeast Petroleum University \\ Da Qing, China \\ Email: wangwei050802@163.com \\ Email: hejinbao2004@163.com
}

\author{
Wang Huzhen \\ Northeast Petroleum University \\ Da Qing, China \\ Email: whz1981@163.com
}

\begin{abstract}
In this article, the neural network method is applied to optimize repeated acidizing fracturing Wells. In order to obtain accurate prediction results reverse passing through error and adjust the weights of each layer are adopt. This method applied in Zanarol oil field has got a good effect.
\end{abstract}

Keywords- Neural network;acid fracturing; Selected; optimise; carbonatite

\section{INTRODUCTION}

Zanarol oil field is in the right south $240 \mathrm{~km}$ of the Aqtobe in the Republic of Kazakhstan. On the regional structure of marina Caspian Sea on the east edge of the basin uplift "a salt under Paleozoic uplift, Oil and gas fields are a set of carboniferous carbonate rock reservoir. Since 2000, Acidizing fracturing technology is widely applied in developing oil field. And has been playing a main role in carbonate gas or oil reservoir stimulation. But acidizing fracturing technology commonly has a definite validity. And relative to the sand fracturing, the validity is usually shorter. Usually quadratic transformation is applied to restore the single well production capacity for failure Wells. So the study against acid fracturing of choosing wells and lays has played a crucial role in improving the effect of modification.

In recent years, International technical services company has carried out a great deal of field experiments and theoretical studies against carbonate reservoir of the key technology of choosing wells and layers, especially direct at the most important method of choosing wells and layers, while the study at this method, Advanced Resources International company, Schlumberger, Intelligent Solutions company, Ely and Associates company, Stem-Lab and Pinnacle technology company etc. have all carried out a large amount of research work. This study has closely combined complex carbonate oil field actual situation overseas, based on the studies of the literature domestic and abroad, Using artificial neural network method, and predicting the well productivity combining with characteristic of the reservoir, So as to realize the carbonate repeated optimization of acid fracturing modification candidate Wells.

Due to the relationship between fracturing effect and its parameters are mostly complex nonlinear. This relationship is difficult to express using traditional mathematical. And due to the different influence degree of each factor on the fracturing effect, and some factors have the phenomenon of crossing influences. Thus of artificial neural network method can be used to solve this kind of highly complicated nonlinear problem. In dealing with this complex nonlinear relationship between the parameters, through the neuron's parameters (the complicated network structure) on the Internet to express knowledge rather than using simple expressions in traditional mathematics. In order to solve the phenomenon of repetition and primary and secondary between parameters by adjusting the network of each node and the connection between the coefficients (the size of the parameters weights).

\section{THE BASIC PRINCIPLE OF ARTIFICIAL NEURAL NETWORK}

Neural network is a kind of network that consist of using computer hardware or software to simulate the brain networks of neurons and nerve fibers to form a knowledge of complex phenomena. This cognition is not expressed in a simple formula, but in the network on each neuron's parameters and the weight coefficient of fiber, it's using is divided into two stages of learning and application step. In the learning step, users only need to present both a great deal of test data and conclusions (and the result data) to the network. It will complete the learning process automatically.

Adjusting the parameters of the neural network so as to give the correct response to the given data. Since networks are built, it can enter into the application stage, at this time it can deal with the new data, to obtain some results of correct response, and it can also learn something in the process of application, the processing precision of the network depends on the past several experiences gained from the previous learning. Artificial neural network can be used to deal with extremely complex relationships because 
of the artificial neural network express knowledge with complex network structure that composed of a large number of neurons. Especially for those phenomenon that have no
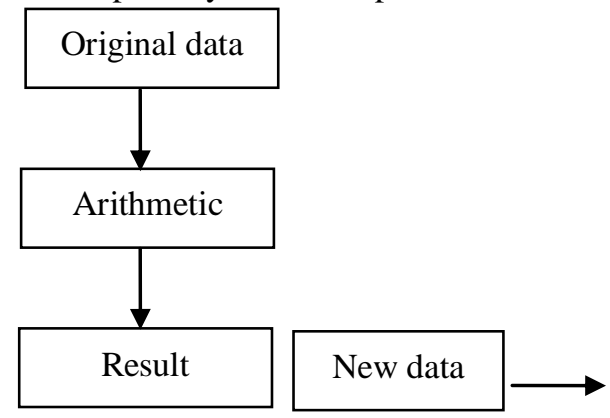

a. traditional method obvious regularity. The Figure 1 below is the comparison with artificial neural network and the traditional method.

Figure 1. Comparison of traditional method and artificial neural network method

The main characteristic of artificial neural network:

a) Neural network can distribute storing information's information is not stored in a single place but is distributed in different places. A portion of the network does not only store a message, but store in step by step. Even the local network is damaged; the original information can also be restored.

b) The processing and reasoning process of information can be done in the same time.

c) Neural network can process information in the way of self-organizing and self-learning rather than studying and summarizing the regulation of the data in advance. Neurons are the basic component unit of artificial neural network. Each neuron constitutes a complicated network (neural network) in the three-dimensional space. According to the connection pattern of neurons in the neural network, neural network can be divided into two basic types: layered network and mesh network.

\section{MATHEMATICAL MODEL OF ARTIFICIAL NEURAL NETWORK}

BP neural network include of input layer, interface layer (hidden layer) and output layer. It is connection between the up and down level, while there is no connection between each layer of neurons. After a pair of learning samples is provided to network, the activation values of neuron transmit from the input layer through each layer to the output layer and each neuron network in the output layer gains the input response. According to reduce the target output and the actual error direction, amending each connection weight from the output layer to each layer correction, step by step, and finally returned to the input layer. The accuracy of the impact of network on the input mode has increased with amending the error constantly. And a basic neural model should include $\mathrm{R}$ inputs and each input connects to neurons through an appropriate weight.

The BP neural network is composed of input layer, hidden layer and output layer, and own n, m, 1 neurons respectively, as shown in Figure 2.

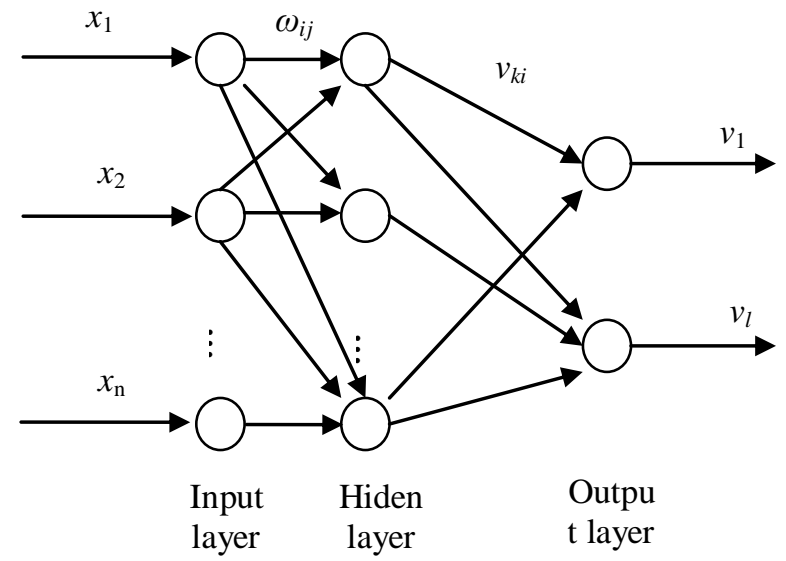

Figure 2. Neural network model

It has $P$ training samples, setting the input of the pth $(p=1,2, \ldots, P)$ sample is $x(p)=\left(x_{1}(p), x_{2}(p), \ldots, x_{\mathrm{n}}(p)\right)$, it's actual output is $t(p)=\left(t_{1}(p), t_{2}(p), \ldots, t_{1}(p)\right)$, the network output is $y(p)=\left(y_{1}(p), y_{2}(p), \ldots, y_{1}(p)\right)$. The training steps are as follows:

\section{A. Positive-going Calculation}

Calculate the input $I_{i p}$ of each neuron in hidden layer.

$$
I_{i p}=\sum_{j=1}^{n}\left(w_{i j} x_{j p}\right)+\theta_{i}
$$

The output $O_{i p}$ of neurons in hidden layer.

$$
O_{i p}=f\left(I_{i p}\right)=\frac{1}{a+b e^{-c I_{i p}}}
$$

The output $y_{k p}$ of neurons in output layer.

$$
y_{k p}=\sum_{i=1}^{m}\left(v_{k i} O_{i p}\right)
$$

Where:

$w_{i j}$ the bottom-up weight of hidden layer neuron 
$i(i=1,2, \ldots, m)$ and input layer node $j$;

$x_{j p}$ - the $\mathrm{j}$ input value of the $\mathrm{p}$ sample;

$\theta_{i}$ - the biases of hidden neuron $i$;

$f$ - the excitation function of hidden neuron;

$a, b, c \_$adjustable parameters, generally 1 ;

$v_{k i}$ the bottom-up weight of output layer neuron $k(k=1,2, \ldots, l)$ and hidden layer neuron $i$.

\section{B. Back Propagation Algorithm}

We define network output error $\mathrm{dp}$ and sample error function ep as follow respectively:

$$
\begin{gathered}
d_{p}=t_{k p}-y_{k p} \\
e_{p}=\frac{1}{2} \sum_{k=1}^{l}\left(t_{k p}-y_{k p}\right)^{2}
\end{gathered}
$$

Where:

$t_{k p}$ the $k$ actual output value of the $p$ sample;

$y_{k p}$ - the $k$ network output value of the $p$ sample.

Let $W=[w, \theta, v]$, the correction of $W: \Delta W=\left[\Delta w_{i j}, \Delta \theta_{i}, \Delta v_{i}\right]$, in the back-propagation algorithm, along with the direction of the negative gradient of the error function $e_{p}$, changing with $W, \Delta W$ revise $W$.

$$
\Delta W^{(p)}=-\eta \frac{\partial e_{p}}{\partial W}+\alpha \Delta W^{(p-1)}
$$

Where:

$\eta$-learning rate, the range is $[0,1]$, generally $0.02 \sim 0.3$;

$\alpha-$ momentum factor, the range is $[0,1]$, generally $0.30 \sim 0.95$.

$$
\begin{gathered}
\Delta v_{i}=\eta d_{p} \frac{\partial y_{p}}{\partial v_{i}}=\eta d_{p} O_{i p} \\
\Delta \theta_{i}=\eta d_{p} \frac{\partial y_{p}}{\partial q_{i}}=c \eta d_{p} v_{i} O_{i p}\left(1-a O_{i p}\right) \\
\Delta w_{i j}=\eta d_{p} \frac{\partial y_{p}}{\partial w_{i j}}=c \eta d_{p} v_{i} O_{i p}\left(1-a O_{i p}\right) x_{j p}
\end{gathered}
$$

$W+\Delta W \rightarrow W$ is used to revise original bottom-up weight vector $W$ into forward calculation of learning sample $p+1$.
Energy function $E$ after finishing training of $P$ learning samples is defined as:

$$
E=\sum_{p=1}^{p} e_{p}=\frac{1}{2} \sum_{p=1}^{p}\left(t_{p}-y_{p}\right)^{2}
$$

If the value of $\mathrm{E}$ can meet the demand of some precision value (training error), then stop iteration, or continue another new round iterative calculation.

\section{BP ALGORITHM STEPS}

The steps of BP algorithm are shown in Figure 3, as follows:

The first step, the network initialization, the very bottom-up weight is assigned to a random number of interval of $(1,1)$, the error function e is set, the calculation precision value $\xi$ and maximum learning times $M$ are determined.

The second step, the $\mathrm{k}$ input sample and the corresponding desirable output are randomly selected.

The third step, the input Iip and output Oip of each hidden layer neuron are calculated.

The fourth step, the network desirable output and actual output are used to calculate partial derivatives of the error function to the output layer neurons.

The fifth step, the bottom-up weight of the hidden layer to the output layer, partial derivatives of the output layer neurons and output of the hidden layer are used to calculate partial derivatives of the error function to the hidden layer neurons.

The sixth step, partial derivatives of the output layer neurons and output of the hidden layer neurons are used to revise the bottom-up weight.

The seventh step, partial derivatives of the hidden layer neurons and input of the output layer neurons are used to revise the bottom-up weight.

The eighth step, a global error $\mathrm{E}$ is calculated.

The ninth step, we judge whether the network error can meet the precision demand. When the error reaches the preset precision value or learning times is larger than the set maximum $\mathrm{M}$, then the algorithm terminates. Otherwise, next learning sample and corresponding desirable output are selected, and return to the third step, and continue next round learning. 


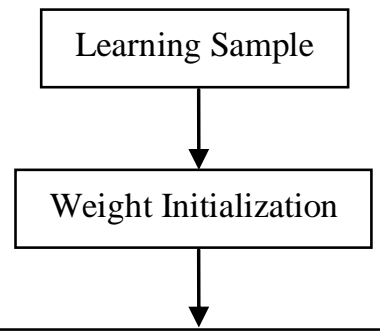

Forward Propagation to Calculate Output Values of the Hidden and Output Layer Neurons

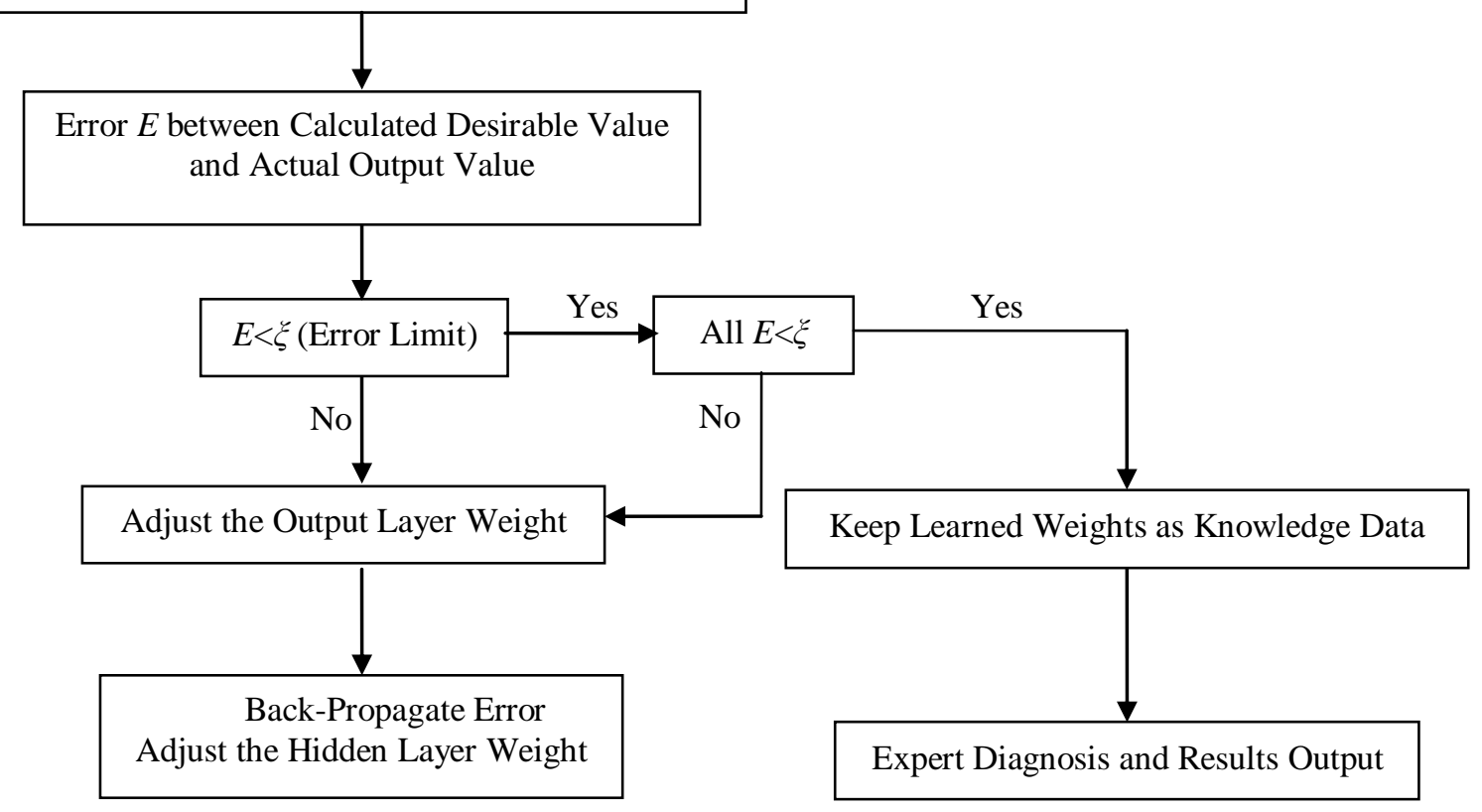

Figure 3. Learning Diagram of BP Algorithm

\section{ACTUAL APPLICATION}

For 6 acid fracturing wells of Zanarol Oilfield, yield prediction of 6 wells is conducted (Table 1), permeability, porosity, oil saturation, effective thickness, acid dosage are selected, the current yield is considered as input sample, the prediction yield after fracturing is considered as output sample. The results show that the prediction results of artificial neural network and the actual production data are basically consistent, achieving good prediction effect.

TABLE I. YIELD PREDICTION AFTER ACID REFRACTURING TREATMENT OF ZANAROL OILFIELD

\begin{tabular}{|c|c|c|c|c|c|c|c|}
\hline Well NO. & $\begin{array}{l}\text { Permeability } \\
\left(10^{-15} \mathrm{~m}^{2}\right)\end{array}$ & $\begin{array}{c}\text { Porosity } \\
(\%)\end{array}$ & $\begin{array}{c}\text { Oil Saturation } \\
(\%)\end{array}$ & $\begin{array}{c}\text { Effective } \\
\text { Thickness (m) }\end{array}$ & $\begin{array}{c}\text { Acid } \\
\text { Dosage }\left(\mathrm{m}^{3}\right)\end{array}$ & $\begin{array}{l}\text { Current Yield } \\
(\mathrm{t} / \mathrm{d})\end{array}$ & $\begin{array}{c}\text { Yield } \\
\text { Prediction } \\
\text { (t/d) }\end{array}$ \\
\hline 2092 & 2 & 12 & 76 & 65 & 234 & 3 & 54.42 \\
\hline 2449 & 6 & 12.03 & 72.72 & 44 & 266.5 & 13 & 73.78 \\
\hline 3324 & 29.41 & 8.79 & 78.98 & 16.6 & 210 & 8 & 73.03 \\
\hline 3477 & 8 & 11.02 & 78 & 8 & 116 & 6 & 9.86 \\
\hline 3561 & 14.33 & 10.78 & 87.39 & 37.6 & 221.3 & 17 & 22.75 \\
\hline 3563 & 8.99 & 10.07 & 79.13 & 21.2 & 228 & 14 & 21.78 \\
\hline
\end{tabular}

\section{REFERENCES}

[1] Roshanai Heydarabadi ,J. Moghadasi, Criteria for Selecting a Candidate Well for Hydraulic Fracturing[C],SPE 136988 presented at the Nigeria Annual International Conference and Exhibition, held in Tinapa - Calabar, Nigeria.31 July - 7 August 2010.
[2] Benson Oghenovo Ugbenyen, Samuel O. Osisanya, Efficient Methodology for Stimulation Candidate Selection and Well Workover Optimization[C],SPE 150760 presented at the Nigeria Annual International Conference and Exhibition,held in Abuja, Nigeria .30 July - 3 August 2011.

[3] Mansoor Zoveidavianpoor, Ariffin Samsuri, Hydraulic Fracturing 
Candidate-Well Selection by Interval Type-2 Fuzzy Set and System[C],SPE 16615 presented at the 6th International Petroleum Technology Conference, held in Beijing, China. 26 - 28 Mar 2013.

[4] Cao Dinghong, Ni Yuwei, Application and Realization of Fuzzy Method for Selecting Wells and Formations in Fracturing in Putaohua Oilfield: Production and Operations: Diagnostics and Evaluation[C],SPE 106335 presented at the SPE Technical Symposium of Saudi Arabia Section, held in Dhahran, Saudi Arabia.21-23 May 2006.

[5] Mohaghegh, S, Platon, V, Candidate Selection for Stimulation of Gas Storage Wells Using Available Data With Neural Networks and Genetic Algorithms[C],SPE 51080 presented at the SPE Eastern
Regional Meeting, Pittsburgh, Pennsylvania, U.S.A.9-11 November 1998.

[6] Shahab Mohaghegh, Scott Reeves, Development of an Intelligent Systems Approach for Restimulation Candidate Selection[C],SPE 59767 presented at the SPE/CERI Gas Technology Symposium, held in Calgary, Alberta, Canada.3-5 April 2000.

[7] S.R. Reeves, Advanced Resources Intl., P.A. Bastian, Benchmarking of Restimulation Candidate Selection Techniques in Layered, Tigh Gas Sand Formations Using Reservoir Simulation[C],SPE 63096 presented at the SPE Annual Technical Conference and Exhibition, held in Dallas, Texas,U.S.A.1-4 October 2000. 